\title{
Planspiele in der ERP-Lehre
}

\section{Eine empirische Untersuchung deutscher Bildungseinrich- tungen}

\author{
Christos Konstantinidis, Harald Kienegger, Holger Wittges, Helmut Kromar \\ Lehrstubl für Wirtschaftsinformatik, \\ Technische Universität München
}

\section{Einleitung}

Der stetige Wandel und Fortschritt im Umfeld betrieblicher Informationssysteme stellen sowohl Unternehmen als auch Bildungseinrichtungen vor neue Herausforderungen. Dabei kommt den Enterprise Resource Planning (ERP) Systemen eine besondere Bedeutung zu. Diese „[...] bilden heutzutage in vielen Unternehmen das Rückgrat der Informationsverarbeitung [...]“" (Gronau 2004) und stellen demnach einen wesentlichen Erfolgsfaktor für Unternehmen dar.

Aus diesem Grund ist es erforderlich, die Verfügbarkeit von qualifiziertem Personal sicherzustellen und ein besonderes Augenmerk auf dessen Ausbildung zu legen (Mohr 2009). Um die Bewältigung sowohl von täglichen Aufgaben als auch das Verständnis für grundlegende Konzepte und Verfahren gewährleisten zu können, ist es notwendig, ein weiterführendes Lehrmodell zu etablieren, das nicht nur mit klassischen Schulungen abschließt. Die Komplexität von ERP-Lösungen erfordert, dass sich die Qualifizierung nicht nur auf konzeptuelles und Handhabungswissen beschränkt, sondern auch den Prozesskontext aufzeigt (Eggert 2005, S. 19).

Konnten diese Tätigkeitsfelder vormals eindeutig der unternehmerischen Fortund Weiterbildung zugeordnet werden, zeigen Entwicklungen der letzten Jahre, dass es zunehmend zu einer Verschiebung in Richtung der Bildungseinrichtungen kommt (Hawking und McCarthy 2000, S. 130-132). Die traditionelle Trennung zwischen der Vermittlung theoretischer Inhalte, als Domäne des tertiären Bildungssektors, und das Erlangen praktischer Erfahrungen im unternehmerischen Umfeld vermischen sich zunehmend.

Es ist demnach erforderlich, insbesondere im Kontext der Wirtschaftsinformatik, sowohl eine praxisnahe als auch theoretisch fundierte Lehre anzubieten, die den Absolventen einerseits konzeptuelles Wissen vermittelt und andererseits den 
Anforderungen der Unternehmen entspricht (Buhl und König 2007, S. 241-242; Frank 2008, S. 41). Dabei kommt es nicht nur auf den Transfer von Faktenwissen an, sondern auch auf die Vermittlung von prozessorientierten Inhalten und von sogenannten Softskills. Traditionelle Lehrmethoden entsprechen diesen Anforderungen meist kaum oder gar nicht.

Es stellt sich demnach die Frage, wie den zukünftigen ERP-Spezialisten eine Ausbildung ermöglicht werden kann, die diesen Anforderungen entspricht. Eine mögliche Antwort hierauf könnten Planspiele darstellen. Denn insbesondere der praktische Ansatz (Learning-by-doing) ermöglicht eine praxisnahe, aber auch nachhaltige Ausbildung und Vermittlung von Lerninhalten.

Ziel dieser Arbeit ist zu ermitteln, inwieweit in der deutschen Hochschullandschaft Planspiele im Rahmen der ERP-Lehre eingesetzt werden und welche aktuellen Möglichkeiten bzw. Trends derzeit bestehen, diese Lehrmethode in der ERPAusbildung anzubieten. Dazu wurden Bildungseinrichtungen untersucht, die sich bereits mit der praktischen ERP-Ausbildung beschäftigt haben und demnach als repräsentativ für die angesprochene Thematik gelten können.

\section{Related Work}

Der Prozess der Wissensvermittlung und dessen Nachhaltigkeit stellt eine Herausforderung sowohl für Unternehmen als auch für Bildungseinrichtungen dar. Dabei wurde der Einsatz von ERP-Systemen in der universitären Lehre bereits in den 1990er Jahren vielfach diskutiert (Gable et al. 1997; Watson et al. 1999; Watson und Schneider 1999). Gründe hierfür waren die steigenden und sich stetig ändernden Anforderungen des Arbeitsmarktes sowie die Notwendigkeit, theoretische Konzepte anhand praktischer Methoden zu vermitteln, was eine Umstrukturierung der Curricula an den Bildungseinrichtungen erforderte (Becerra-Fernandez et al. 2000, S. 39; Stewart et al. 1999). In ihrer Studie zu ERP-Systemen: Enterprise Resource Planning Systems Research - An annotated Bibliography zeigten Esteves und Pastor (2001, S. 31-33) anhand einer empirischen Untersuchung besagte Entwicklung auf, die in ihrer Neuauflage im Jahre 2007 diesen Trend erneut beschrieb (Esteves und Bohórquez 2007, S. 416-419). Ermöglicht wurden diese praktischen Lehrmethoden durch die Zusammenarbeit mit Systemhäusern wie z. B. der SAP oder IBM, die den Studierenden die Möglichkeit boten, Erfahrungen im Umgang mit deren Softwarelösungen zu sammeln.

Heute existieren mehrere Programme ${ }^{1}$, die sich auf diese Zusammenarbeit spezialisiert haben und eine praxisnahe sowie -relevante Ausbildung an unterschiedlichen Bildungseinrichtungen ermöglichen. Die Vorzüge, aber auch Schwierigkeiten, konzeptuelles Wissen mittels ERP-Systemen zu vermitteln, wurden bereits des Öfteren kritisch untersucht (Murphy 2007; Noguera und Watson 2004; Scott

\footnotetext{
${ }^{1}$ Das University Alliances Program der SAP und die IBM University Relations können an dieser Stelle exemplarisch genannt werden.
} 
1999). Dabei erwies sich insbesondere der praktische Umgang mit den Softwarelösungen als effektiv, um theoretisch vermittelte Inhalte nachhaltig zu festigen (Lee et al. 2006, S. 78-81; Maurizio und Rosemann 2005, S. 443-444). Die steigende Anzahl an Institutionen, die den praktischen Umgang mit Informationssystemen anbieten, kann als weiteres Indiz für die Relevanz dieser praktischen Wissensvermittlung interpretiert werden (Corbitt und Mensching 2000; Maurizio und Rosemann 2005, S. 448-449).

Allerdings beruhen alle vorausgegangenen Beobachtungen auf der praktischen Vermittlung von Inhalten mittels Demonstrationen und Fallstudien, wobei ersteres den Studierenden keinen direkten Umgang mit dem System ermöglicht. Der Frontalunterricht stellt dabei noch einen wesentlichen Bestandteil der Wissensvermittlung dar.

Weiterhin wurden E-Learnings im Kontext der ERP-Ausbildung als mögliches Instrument zum Wissenstransfer untersucht (Hawking und McCarthy 2001; McCarthy und Paul 2004). Allerdings stehen hier die geographische und zeitliche Unabhängigkeit der Inhaltsvermittlung im Vordergrund, sowie die Möglichkeit, Lehrinhalte modular bereitzustellen und diese einer möglichst großen Teilnehmerzahl (Skaleneffekte) zu vermitteln (Back et al. 2001; Dittler 2003). Auch hier gilt es zu konstatieren, dass diese Lernmethode dem Lernenden zwar einen komfortablen Zugang zu den Inhalten ermöglicht, praktische Erfahrungen und der Umgang mit ERP-Lösungen jedoch nur schwer zu realisieren sind und demnach eine nachhaltige Wissensvermittlung fragwürdig bleibt.

Nicht alle Lehrmethoden entsprechen den genannten Anforderungen einer nachhaltigen und praxisnahen Ausbildung und stellen demnach keine effiziente Wissensvermittlung in diesem Kontext dar (Adelsberger et al. 1999).

\section{Planspiele}

\subsection{Lehrmethoden zur Wissens- und Kompetenzaneignung}

Verschiedene Lehrmethoden, als angewandtes Konzept zur Wissensvermittlung, werden von Carls und Koeder (1988, S. 6) beschrieben. Danach lassen sich (1) passive und (2) aktive (Lern-/)Lehrformen unterscheiden. Als passive Unterrichtsform ist im tertiären Bildungsbereich vor allem die (1a) Vorlesung bzw. der Frontalunterricht weit verbreitet. Bei den aktiven Lehrformen unterscheidet man (2a) Lehrgespräche sowie (2b) Simulationen. Simulationen können dabei in (i) Fallstudien, (ii) Plan- und (iii) Rollenspiele unterschieden werden.

Keine der dabei aufgeführten Lehrformen stellt jedoch eine One-Size-Fits-AllLösung dar. Vielmehr eignen sich diese, je nach pädagogischer Orientierung, für die Vermittlung unterschiedlichen Wissens und unterschiedlicher Kompetenzen. Im Bereich der Wissensvermittlung können nach Kern (2003, S. 18-23) folgende Formen unterschieden werden: 
1. Sollen theoretisches Basiswissen, Terminologie und (mathematische) Formeln weitergegeben werden, handelt es sich um Faktenwissen (know what).

2. Beim Anwendungswissen (know how) steht das Erlernen bestimmter Fertigkeiten, Methoden und Techniken im Vordergrund, d. h. das zuvor Erlernte aus 1. anzuwenden.

3. Hingegen hat das Vermitteln von Zusammenhangs- und Transferwissen (know why) das Ziel, das zuvor theoretisch Erlernte miteinander zu verknüpfen und z. B. Lösungskonzepte von einer Domäne in eine andere zu übertragen.

4. Softskills wie Team-, Koordinations- und Konfliktlösungsfähigkeiten werden beim Orientierungswissen (care why) forciert.

Für die drei zuletzt genannten Wissensformen eignen sich Planspiele besonders. Beim Faktenwissen sind sie weniger zur Wissensaneignung sondern für Wiederholungen geeignet. Alle vier Kompetenzarten werden für die im beruflichen Umfeld wichtige Qualifikation, die Handlungskompetenz, benötigt. Sie umfasst Fähigkeiten, die es einem Individuum erlauben, sich in beruflichen und anderen (sozialen) Lagen, situationsspezifisch, qualifiziert und sozial verantwortlich zu verhalten.

\subsection{Charakteristika und Definition von Planspielen}

Bereits aus den Worten - Planung und Spiel - die den Begriff bilden, lassen sich wesentliche Eigenschaften für Planspiele ableiten. Nach Kriz (2001, S. 52) werden Planspiele durch folgende drei Merkmale charakterisiert:

1. Das Spiel (im Englischen: Game) wird als System von Regeln verstanden, mit dem Ziel, relevante und vorher festgelegte Prozesse zu strukturieren (Spielcharakter).

2. Dieses (geschlossene) System oder eine definierte Teilmenge davon wird im Verlauf des Spiels simuliert. Dabei können im Rahmen dieser Simulation, die als technische Abbildung eines Modells verstanden werden kann, Systemelemente untereinander interagieren. In der Regel findet allerdings keine Interaktion auBerhalb des Systems statt (Planungscharakter).

3. Im Rahmen des Spiels werden verschiedene Rollen als Funktionen definiert, die in der dynamischen Spielsituation von Personen übernommen werden (Planungs- und Spielcharakter).

Eine ähnliche Charakterisierung führen auch Adelsberger et al. (1999, S. 455) durch, indem sie der Beschreibung (siehe Planungscharakter) und der Simulation (siehe Spiel) von Planspielen deren anschließende Evaluation als zusätzliche Komponente hinzufügen. Insbesondere in der (ERP-)Lehre spielt nach Léger (2007, S. 13) die Evaluation der Spielergebnisse und der -teilnehmer eine Schlüsselrolle. Die erzielten Ergebnisse der Teams bzw. Spielteilnehmer stehen dabei weniger im Vordergrund. Vielmehr sollte überprüft werden, ob das pädagogisch angestrebte Ziel, die Vermittlung der einzelnen Wissensbereiche, insbesondere der praktische Umgang mit dem ERP-System und das Verständnis für die zugrundeliegenden Geschäftsprozesse, erreicht wurde. 
Gemäß obigen Überlegungen lässt sich ein Planspiel als „[... eine konstruierte Situation, in der sich eine oder mehrere Person(en) in oder an einem [...] Modell nach vorgegebenen Regeln verhalten, wobei das gezeigte Verhalten systematisch festgehalten und nach einem explizierbaren Kalkül bewertet werden kann“ (Geilhardt und Mühlbradt 1995, S. 49) definieren.

Die Differenzierung von Planspielen und Simulationen wird in der Literatur kontrovers diskutiert. Eine mögliche Unterscheidung beider Begriffe kann anhand des dynamischen (Rollen-)Verlaufs gemäß obiger Beschreibung erfolgen. Dieser teilt sich nach Kern (2003, S. 77) in zwei Bereiche:

1. Während eines Planspiels führt die handelnde Person, abhängig von der von ihr eingenommenen Rolle, Aktivitäten aus. Daher wird dieser auch Aktionsbereich genannt.

2. Die darauf folgende Simulation verarbeitet die in 1. getätigten Aktivitäten bzw. Eingaben (bei IT-unterstützten Planspielen) entsprechend zu Ergebnissen und wird demnach als Reaktionsbereich bezeichnet.

Abbildung 1 stellt graphisch Aktions- und Reaktionsbereich dar. Dabei wird deutlich, dass Planspiele beide Bereiche abdecken, wohingegen die Simulation primär nur den Reaktionsbereich berücksichtigt.

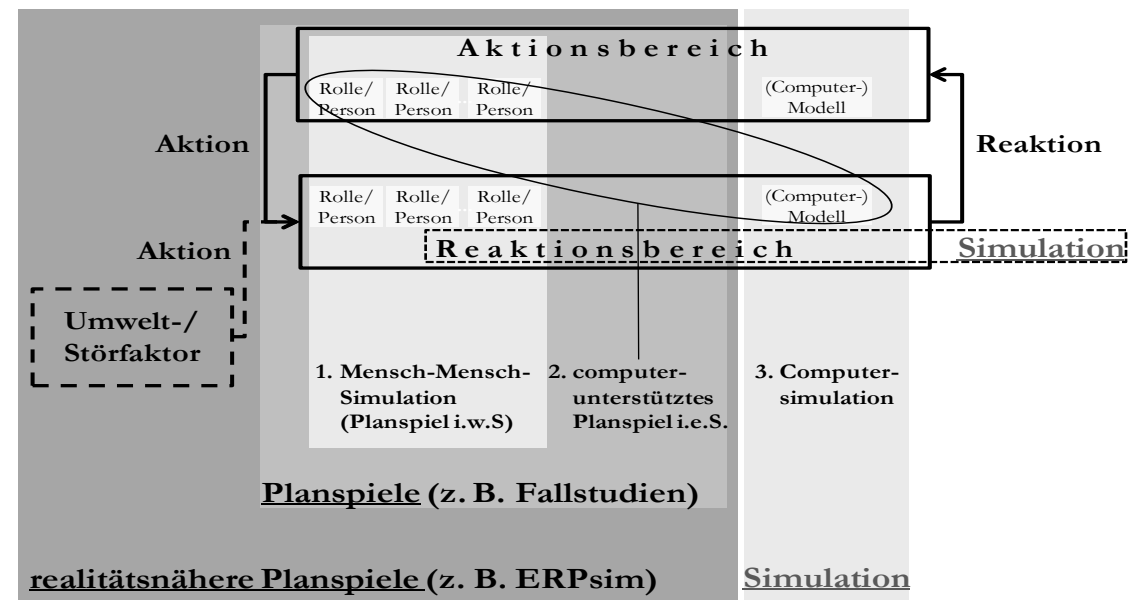

Abbildung 1: Formen von Planspielen und Abgrenzung zur (reinen) Simulation

Neben den Planspielen im weiteren und engeren Sinne, wird an dieser Stelle ein weiteres Klassifikationsmerkmal aufgeführt. Dabei handelt es sich um eine Systemkomponente, die für die Simulation von (unvorhersehbaren) Umweltereignissen zuständig ist. Unter Berücksichtigung dieser Umwelt- oder Störfaktoren kann von sogenannten realitätsnäheren Planspielen, wie in Abbildung 1 dargestellt, gesprochen werden. 


\section{Planspiele in der heutigen (ERP-)Lehre}

\subsection{Klassische Planspiele in der Lehre}

Obwohl Unternehmen bereits seit mehreren Jahren Planspiele zur betrieblichen Fort- und Weiterbildung nutzen, fand diese Methode im tertiären Bildungssektor lange Zeit keine besondere Beachtung (Nissen 2006). Aktuelle Untersuchungen zeigen jedoch einen gegenläufigen Trend auf. So werden mittlerweile zahlreiche Unternehmens- bzw. Managementplanspiele zur Ausbildung der Studierenden an Bildungseinrichtungen genutzt. Das CIO-Planspiel, entwickelt an der Technischen Universität München am Lehrstuhl für Wirtschaftsinformatik, beschäftigt sich beispielsweise mit den Herausforderungen und Aufgaben eines Chief Financial Officers (CIO) im Kontext der Unternehmensführung und -steuerung (Baume et al. 2006). Es greift besonders die komplexen Zusammenhänge von IT- und Unternehmensstrategie auf und bietet Studierenden die Möglichkeit, ohne reales wirtschaftliches Risiko, als Führungskräfte für mehrere simulierte Jahre die Leitung einer Autobank mit hoher IT-Durchdringung zu übernehmen. Durch ein Rollenkonzept werden dabei verschiedene Perspektiven des Top Managements im Unternehmen abgebildet. Weiterhin kann die ERP-Simulation des Technology and Innovation Management (TIM) Program der Universität der Bundeswehr München, basierend auf einer Webapplikation (PPS-Planspiel), kann hier beispielhaft aufgeführt werden.

Grundsätzlich stellen konventionelle computergestützte Planspiele (vgl. hierzu computerunterstützte Planspiele in Abbildung 1) eine eigenständige Softwarelösung dar, die für spezielle Szenarien explizit erstellt bzw. konfiguriert wurden. Zudem ist hervorzuheben, dass betrachtete Lösungen meist nur einzelne Bereiche eines ERP-Systems abbilden (können). Dies ist meist auf die Komplexität dieser betrieblichen Informationssysteme zurückzuführen, die es äußert schwer gestaltet, alle Funktionalitäten eines ERP-Systems samt integrativer Bestandteile mittels einer (Planspiel-)Lösung abzubilden. So fokussiert sich bspw. das oben genannte PPS-Planspiel auf die Kern- und Querschnittsaufgaben der Produktionsplanung und -steuerung. Eine Erweiterung dieses Funktionsumfangs ist meist nur mit einer Anpassung der besagten Software möglich, was wiederum mit einem erheblichen Aufwand verbunden wäre.

\subsection{ERPsim - ein realitätsnäheres Planspiel}

Im Folgenden soll das Planspiel ERPsim und dessen Umfang beschrieben werden. ERPsim wurde an der Hautes Études Commerciales (HEC) Montreal in Kanada im Jahr 2004 entwickelt (Léger et al. 2009). Es besteht aus zwei wesentlichen Komponenten: (1) einem SAP ECC 6.0 Standardsystem und (2) einer für das Planspiel entwickelten Java Webapplikation, die auf einem Webserver betrieben wird und für das (automatische) Initiieren bestimmter Transaktionen im SAP-System 
zuständig ist (Simulation). Darüber hinaus enthält sie auch das (technische) Regelwerk und dient als Auslöser der Umwelt- bzw. Störfaktoren.

Anknüpfend an die in Abbildung 1 vorgenommene Differenzierung zwischen Planspiel und Simulation sollen in Abbildung 2 die Zusammenhänge und Interaktionen der unterschiedlichen Planspielkomponenten aus der Perspektive von ERPsim zusammengefasst werden.

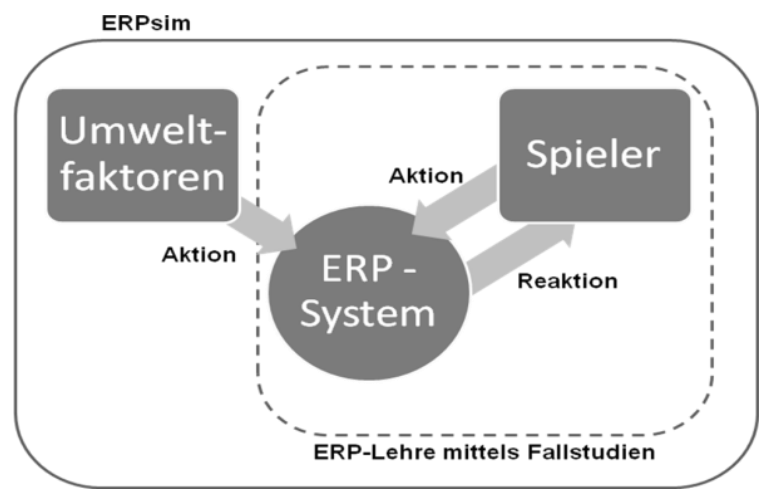

\section{Abbildung 2: Interaktion der unterschiedlichen Komponenten}

Die Spieler können Aktionen direkt auf dem ERP-System ausführen. Dieses reagiert auf die getätigten Eingaben/ Transaktionen und simuliert bereits in der Interkation zwischen Spieler und ERP-System entsprechende Vorgänge (z. B. das Erstellen von Absatz- oder Produktionsplanungen, Materialverfügbarkeitsprüfungen usw.). ERPsim geht dabei auf die Benutzereingaben ein und automatisiert verschiedene Unternehmensprozesse, z. B. das Aufgeben von Kundenaufträgen in Abhängigkeit der vom Benutzer gewählten Produktpreise.

Mit Hilfe des Planspiels ERPsim können somit in der ERP-Lehre folgende Prozesse vollständig oder teilweise abgedeckt werden: Planung, Marketing, Beschaffung, Fertigung, Verkauf, Finanzen und Controlling. Dabei automatisiert die oben vorgestellte Java Webapplikation folgende Geschäftsprozesse (vgl. Abbildung 3): (1) die Nachfrage, damit jedes Unternehmen eine gewisse Anzahl an Aufträgen in jeder Periode zur Verfügung stehen hat, (2) einen Teil der Produktion, um Kapazitätsbeschränkungen während der Fertigung abzubilden und (3) einen Teil der Beschaffung, um Verzögerungen bei Lieferung zu simulieren. Mit Hilfe von Standard- und kundenspezifischen SAP-Berichten, müssen die Studierenden ihre Daten analysieren, um unternehmerische Entscheidungen zu treffen und die Wirtschaftlichkeit ihrer Geschäftstätigkeiten sicherzustellen.

Die Spieler erlernen mit Hilfe von ERPsim, die Zusammenhänge zwischen getroffenen Entscheidungen und den daraus resultierenden Ergebnissen zu verstehen und das zuvor theoretisch erlangte (konzeptuelle) Wissen, mit Hilfe praktischer Übungen zu festigen bzw. umzusetzen. Somit eignet sich ERPsim einerseits zur Verdeutlichung von Faktenwissen und der betriebswirtschaftlich zugrundeliegenden Geschäftsprozessen bzw. deren praktische Umsetzung, andererseits zur Ver- 
mittlung von Softskills und somit Sozialkompetenz aufgrund der Teamarbeit und der rollenbasierten Gestaltung des Spiels.

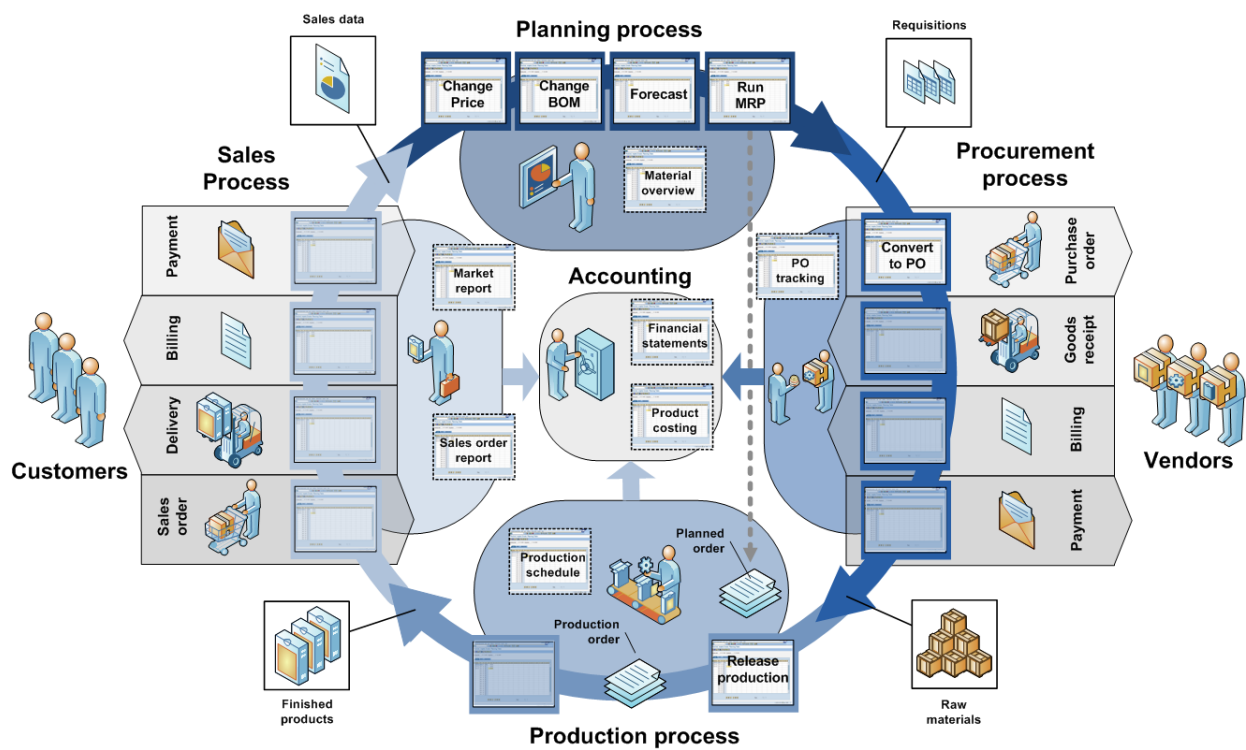

Abbildung 3: Prozesse und Vorgänge bei ERPsim (ERPsim L@b 2009)

Allerdings ist bei einer kritischen Betrachtung festzuhalten, dass auch im Fall von ERPsim nicht das gesamte Spektrum eines ERP-Systems abdeckt wird. Diese Restriktionen können jedoch umgangen werden, da die Nutzer nicht, wie im oben aufgeführten PPS-Planspiel, mit einer eigens für das Spiel erstellten Software arbeiten, sondern sämtliche Aktionen auf einem (realen) ERP-System ausführen. Sie sind demnach nicht zwingend auf die Umwelt- oder Einflussfaktoren der Simulationskomponente angewiesen, sondern könnten auch ohne diese, ein praktisches Wissen im Umgang mit der ERP-Lösung erlangen. So könnten beispielsweise Fallstudien bzw. selbst erstellte Szenarien aus den Bereichen Human Capital Management, Controlling oder Finanzbuchhaltung sinnvolle Ergänzungen zum eigentlichen Planspiel (ERPsim) darstellen. Den Teilnehmern könnte so ein erweitertes Spektrum an Funktionalitäten und Zusammenhängen (innerhalb eines ERPSystems) aufgezeigt werden, was primär das integrative Verständnis erweitern würde.

\section{$5 \quad$ Ergebnisse einer empirischen Untersuchung}

Um zu ermitteln, wie viele Planspiele eingesetzt und in welchem Umfang diese in der Lehre genutzt werden, wurde im September 2009 eine empirische Untersuchung durchgeführt. Der Fokus dieser Untersuchung lag dabei auf den deutschen Bildungseinrichtungen (BE), die an dem SAP University Alliances Program (UAP) 
teilnehmen und ERP-Systeme in der Lehre einsetzen. Dabei beträgt $n=254$ BE, die entweder am SAP University Competence Center (UCC) der Technischen Universität München oder der Universität Magdeburg, den beiden zuständigen Application und Education Service Providern des SAP UAP, angeschlossen sind. Im Einsatz befinden sich unterschiedliche betriebliche Informationssysteme der SAP AG, unter anderem das SAP ERP ab der Version R/3.

Betrachtet wurden Lehrstühle wirtschaftwissenschaftlicher Fakultäten und Studiengänge sowie der Wirtschaftsinformatik. Die BE gliedern sich in Universitäten $(22,83 \%)$, Fachhochschulen (42,91\%), Berufsakademien (7,48\%), Schulen $(19,69 \%)$ sowie sonstige BE $(7,09 \%)$. Untersuchungsgegenstand waren die seit 2004 von den BE angebotenen Planspielen gemäß der Definition in Abschnitt 3.2. Grundlage der Untersuchung waren die auf den Internetseiten der Institutionen angebotenen Lehrveranstaltungen, die inhaltlich bewertet und anschließend entsprechend erfasst wurden.

Von den 254 untersuchten BE wurden in den letzten fünf Jahren 254 Planspiele angeboten ${ }^{2}$. Wurde ein Planspiel bzw. die dazugehörige Veranstaltung (einer BE) innerhalb dieses Zeitraums mehrfach angeboten, wurde dieses nur einmal gewertet. Wie in Abbildung 4 dargestellt, bieten 53,94\% (Summe der BE mit einem Planspielangebot $>0$ ) der untersuchten BE Planspiele in der Lehre an.

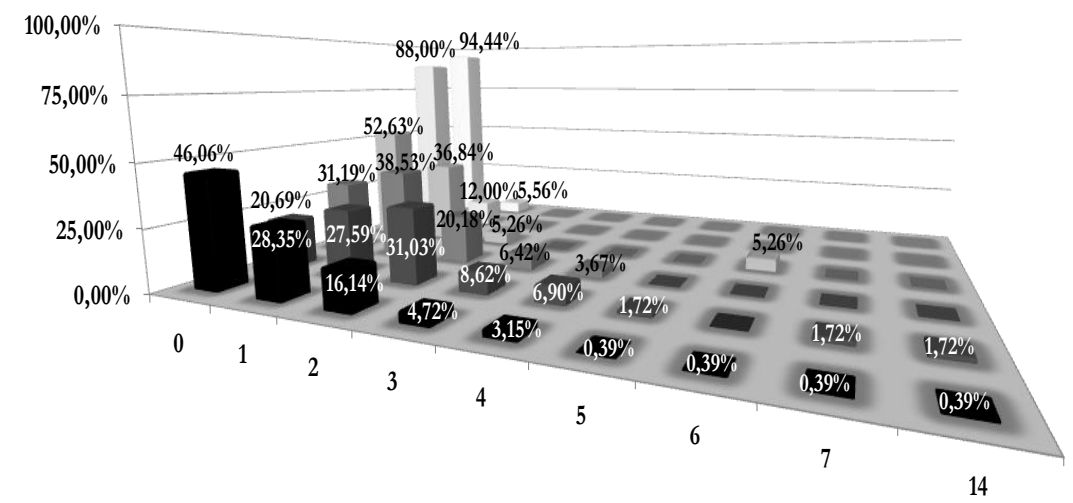

a Grundgesamtheit $(\emptyset=1) \quad \square \mathrm{UNI}(\emptyset=1,88) \quad \square \mathrm{FH}(\emptyset=1,13) \square \mathrm{BA}(\emptyset=0,79) \quad \square$ Schulen $(\emptyset=0,12) \square$ Sonstige $(\emptyset=0,06)$ Abbildung 4: Prozentuale Verteilung angebotener Planspiele je BE

Mehr als die Hälfte dieser BE (28,35\%) boten innerhalb der letzten fünf Jahre mindestens ein Planspiel in ihrem Curriculum an. Über $9 \%$ der betrachteten BE hatten vier oder mehr Planspiele im Einsatz. Betrachtet man die einzelnen BE, so erkennt man bei Universitäten und Fachhochschulen einen ähnlichen Verlauf. Bei beiden ist festzustellen, dass Institutionen, die keine Planspiele anbieten, gegenüber der Grundgesamtheit unterproportional vertreten sind - bei Universitäten 20,69 \% und Fachhochschulen 31,19\%. Dies ist dahingehend interessant, da insbesondere

2 Die Übereinstimmung der Anzahl von Planspielen (= 254) sowie der Bildungseinrichtungen (= 254) ist reiner Zufall und hat keine besondere Bedeutung. 
Universitäten oftmals mit dem Ruf zu kämpfen haben, zu theoretisch und wenig praxisorientiert auszubilden. Dabei zeigt die Untersuchung auf, dass Universitäten gegenüber Fachhochschulen stärker in den Kategorien mit mehreren angebotenen Planspielen vertreten sind. Bieten noch 38,53\% der betrachteten Fachhochschulen, gegenüber $27,59 \%$ bei Universitäten, Planspiele an, so ist eine deutliche Trendwende bei einem Angebot von $>1$ zu erkennen. Über $31 \%$ der untersuchten Universitäten bieten mindestens zwei Planspiele an und knapp 9\% sogar drei. Hervorzuheben sind die in der Grundgesamtheit ermittelten BE, die sieben (Technische Universität Dortmund) oder gar 14 Planspiele (Universität Münster) anbieten. Beide Institutionen entstammen der Gruppe der Universitäten und machen dort jeweils knapp über 1,7\% der BE aus, bieten dabei jedoch 19,27\% der Universitätsplanspiele an. Dieses Ergebnis lässt darauf schließen, dass insbesondere die Universitäten die Möglichkeiten zur Wissensvermittlung mittels Planspielen erkannt und erfolgreich umgesetzt haben.

Ein gegenläufiger Trend zeichnet sich bei den Berufsakademien, Schulen und sonstigen BE ab. Bieten noch knapp $47 \%$ der Berufsakademien mindestens ein Planspiel an, sind die Schulen mit ca. $12 \%$ und sonstigen BE mit etwa $6 \%$ nur gering vertreten.

Auch ein Blick auf die durchschnittlich angebotenen Planspiele pro BE (vgl. hierzu Abbildung 4) verdeutlicht, dass mit knapp zwei Planspielen pro BE der Durchschnitt bei Universitäten, verglichen zu dem der Grundgesamtheit, fast doppelt so hoch ist und auch generell den höchsten Wert darstellt. Lediglich Fachhochschulen liegen mit 1,13 Planspielen pro BE knapp über dem Durchschnitt der Grundgesamtheit.

\section{$6 \quad$ Fazit und Ausblick}

Die Untersuchung hat gezeigt, dass Planspiele bereits eine hohe Durchdringung in der Lehre tertiärer Bildungseinrichtungen erreicht haben. Sie hat jedoch auch gezeigt, dass fast ausschließlich Planspiele im engeren bzw. weiteren Sinne eingesetzt werden. Realitätsnähere Planspiele, wie in Abschnitt 3.2 beschrieben, finden hingegen kaum Anwendung. Obwohl ein derartiges Produkt bereits existiert, wird diese Form der Ausbildung an ERP-Systemen bislang nicht oder nur kaum genutzt wird. Dies ist insbesondere im Hinblick auf deren Möglichkeiten (Erweiterbarkeit, Vermittlung von Softskills etc.) überraschend und die hierfür verantwortlichen Gründe sollten in weiterführenden Untersuchungen genauer betrachtet werden. Eine mögliche Antwort könnte die bislang fehlende Außenwirkung dieser Produkte sein. Mit Hilfe des SAP University Alliances Program besteht nun die Möglichkeit, auf derartige Lehrmethoden zurückgreifen zu können. Dabei müssen die einzelnen Institutionen selbst entscheiden, welche Methode für sie am geeignetsten ist. Denn auch hier gilt: Planspiele stellen zwar eine weitreichende und praxisnahe Ausbildung dar, sind jedoch nicht für alle Arten der Wissensvermittlung geeignet. 
Zudem wurde aufgezeigt, dass der Begriff Planspiel für eine Vielzahl an unterschiedlichen Lehrmethoden stehen kann. Insbesondere in der ERP-Lehre lassen sich hier die Fallstudien einordnen, die den Teilnehmern die Möglichkeit geben, mit einer praktischen ERP-Lösung arbeiten zu können. Diese Methode der Inhaltsvermittlung wird besonders im Kontext der praktischen Ausbildung am System verwendet und stellt eine gute Möglichkeit dar, Anwendungswissen zu vermitteln. Allerdings lässt sich dieser Art der Wissensvermittlung, wie am Beispiel von ERPsim gezeigt, sinnvoll erweitern. Der Einfluss von Umweltfaktoren, auf die Spieler reagieren müssen, stellt eine neue Herausforderung dar und ermöglicht somit, neben dem Anwendungswissen, auch die Vermittlung weiterführender Fähigkeiten. So können die einzelnen Teilnehmer im Team agieren und unterschiedliche Rollen/Positionen einnehmen und direkt die Auswirkungen ihrer Entscheidungen nachvollziehen. Dabei interagieren sie zum einen miteinander und lernen, im Team Entscheidungen zu treffen, und zum anderen werden komplexe Sachverhalte mittels einer realen ERP-Lösung vermittelt.

Darüber hinaus ist die Ausbildung an praktischen Systemen sehr von der Qualifikation der einzelnen Dozenten abhängig. Auch im Kontext von Planspielen ist es demnach erforderlich, auf ausreichend qualifizierte Mitarbeiter zurückgreifen zu können, die einerseits über gutes konzeptuelles und Faktenwissen verfügen und andererseits ein Verständnis für interaktive Lehre mit sich bringen.

\section{Literatur}

Adelsberger HH, Bick MH, Kraus U, Pawlowski JM (1999) A simulation game approach for efficient education in enterprise resource planning systems. In: European Simulation Multiconference, Warschau.

Back A, Bendel O, Stoller-Schai D (2001) E-Learning im Unternehmen: Grundlagen - Strategien - Methoden - Technologien. Orell Füssli, Zürich.

Baume M, Häberle O, Krcmar H (2006) Learn to decide and communicate like an IT manager - designing a business game for IS education. In: Americas Conference on Information Systems, Acapulco.

Becerra-Fernandez I, Murphy KE, Simon SJ (2000) Enterprise resource planning: integrating ERP in the business school curriculum. Communications of the ACM 43 (4):39-41.

Buhl HU, König W (2007) Herausforderungen der Globalisierung für die Wirtschaftsinformatik-Ausbildung. WIRTSCHAFTSINFORMATIK 49 (4):241-243.

Carls CU, Koeder KW (1988) Aktives Lernen durch Planspieleinsatz. Winklers Flügelstift 3:5-14. 
Corbitt G, Mensching J (2000) Integrating SAP R/3 into a college of business curriculum - lessons learned. Information Technology and Management 1 (4):247-258.

Dittler U (2003) E-Learning in der betrieblichen Aus- und Weiterbildung. In: Dittler U (Hrsg) E-Learning - Erfolgsfaktoren und Einsatzkonzepte des Lernens mit interaktiven Medien. Oldenbourg, München.

Eggert S (2005) ERP-Qualifizierung. ERP Management 2:19.

ERPsim L@b (2009) The manufactoring game.

http://erpsim.hec.ca/learning/\#/material/13. Abruf am 2009-11-20.

Esteves J, Bohórquez VW (2007) An updated ERP systems annotated

bibliography: 2001-2005. Communications of the Association for Information Systems 19:386-446.

Esteves J, Pastor J (2001) Enterprise resource planning systems research: an annotated bibliography. Communications of the Association for Information Systems 7 (8):1-52.

Frank U (2008) Herausforderungen der Wirtschaftsinformatik in Zeiten des Wandels. In: Jung R, Myrach T (Hrsg) Quo vadis Wirtschaftsinformatik? Gabler, Wiesbaden.

Gable GG, van Den Heever R, Erlank S, Scott J (1997) Using large packaged software in teaching - The case of SAP R/3. In: AIS American Conference, Indianapolis.

Geilhardt T, Mühlbradt T (1995) Planspiele im Personalmanagement und Organisationsmanagement. Hogrefe, Göttingen.

Gronau N (2004) Enterprise Resource Planning und Supply Chain Management. Oldenbourg, München.

Hawking P, McCarthy B (2000) Industry collaboration: a practical approach for ERP education. In: Proceedings of the Australasian conference on Computing education, Melbourne.

Hawking P, McCarthy B (2001) The ERP elearning model for the delivery of ERP (SAP R/3) curriculum into the asian region. In: Informing Science Conference, Krakau.

Kern M (2003) Planspiele im Internet - Netzbasierte Lernarrangements zur Vermittlung betriebswirtschaftlicher Kompetenz. DUV, Wiesbaden.

Kriz WC (2001) Die Planspielmethode als Lernumgebung. In: Mandl H, Keller C, Reiserer M, Geier B (Hrsg) Planspiele im Internet: Konzepte und Praxisbeispiele für den Einsatz in Aus- und Weiterbildung. wbv, Bielefeld. 
Lee H, Chen KL, Yang J (2006) Teaching enterprise resource planning (ERP) systems in the supply chain management course. Communications of The IIMA 6 (3):77-86.

Léger P-M (2007) Using a simulation game approach to teach enterprise resource planning concepts. Journal of Information Systems Education 17 (4):441-447.

Léger P-M, Robert J, Babin G, Pellerin R, Wagner B (2009) Distribution game version 2009-2010. http://erpsim.hec.ca/learning/\#/material/14. Abruf am 2009-11-20.

Maurizio AA, Rosemann M (2005) SAP-related education - status quo and experiences. Journal of Information Systems Education 16 (4):437-453.

McCarthy B, Paul H (2004) ERP e-learning - if you can't take mohammed to the classroom, take the classroom to mohammed. In: Austalasian Conference on Information Systems.

Mohr M (2009) Qualifizierungsstrategien für betriebswirtschaftliche Unternehmenssoftware. Gabler, Wiesbaden.

Murphy KE (2007) Critical cuccess factors for implementing ERP systems as a vehicle for business curriculum integration at large state university. In: Targowski AS, Tarn JM (Hrsg) Enterprise Systems Education in the 21st Century. Information Science Publishing, Hershey.

Nissen S (2006) Das Planspiel in der universitären Lehre. Soziologie 35 (4):468479.

Noguera JH, Watson E (2004) Effectiveness of using an enterprise system to teach process-centered concepts in business education. Journal of Enterprise Information Management 17 (1):56-74.

Scott J (1999) ERP effectiveness in the classroom - assessing congruence with theoretical learning models. In: Americas Conference on Information Systems, Milwaukee.

Stewart G, Gable G, Andrews R, Rosemann M, Chan T (1999) Lessons from the field - a reflection on teaching SAP R/3 and ERP implementation issues. Americas Conference on Information Systems.

Watson E, Rosemann M, Stewart G (1999) An overview of teaching and research using SAP R/3. In: Americas Conference on Information Systems, Milwaukee.

Watson E, Schneider H (1999) Using ERP systems in education. Communication of AIS 1 (2):1-48. 